\title{
Nonlinear polarization rotation in semiconductor optical amplifiers : theory and application to all-optical flip-flop memories
}

\section{Citation for published version (APA):}

Dorren, H. J. S., Lenstra, D., Liu, Y., Hill, M. T., \& Khoe, G. D. (2003). Nonlinear polarization rotation in semiconductor optical amplifiers : theory and application to all-optical flip-flop memories. IEEE Journal of Quantum Electronics, 39(1), 141-148. https://doi.org/10.1109/JQE.2002.806200

DOI:

10.1109/JQE.2002.806200

Document status and date:

Published: 01/01/2003

\section{Document Version:}

Publisher's PDF, also known as Version of Record (includes final page, issue and volume numbers)

\section{Please check the document version of this publication:}

- A submitted manuscript is the version of the article upon submission and before peer-review. There can be important differences between the submitted version and the official published version of record. People interested in the research are advised to contact the author for the final version of the publication, or visit the DOI to the publisher's website.

- The final author version and the galley proof are versions of the publication after peer review.

- The final published version features the final layout of the paper including the volume, issue and page numbers.

Link to publication

\footnotetext{
General rights

- You may freely distribute the URL identifying the publication in the public portal. follow below link for the End User Agreement:

www.tue.nl/taverne

\section{Take down policy}

If you believe that this document breaches copyright please contact us at:

openaccess@tue.nl

providing details and we will investigate your claim.
}

Copyright and moral rights for the publications made accessible in the public portal are retained by the authors and/or other copyright owners and it is a condition of accessing publications that users recognise and abide by the legal requirements associated with these rights.

- Users may download and print one copy of any publication from the public portal for the purpose of private study or research.

- You may not further distribute the material or use it for any profit-making activity or commercial gain

If the publication is distributed under the terms of Article $25 \mathrm{fa}$ of the Dutch Copyright Act, indicated by the "Taverne" license above, please 


\title{
Nonlinear Polarization Rotation in Semiconductor Optical Amplifiers: Theory and Application to All-Optical Flip-Flop Memories
}

\author{
H. J. S. Dorren, Daan Lenstra, Member, IEEE, Yong Liu, Student Member, IEEE, Martin T. Hill, Member, IEEE, and \\ Giok-Djan Khoe, Fellow, IEEE
}

\begin{abstract}
We present a model for polarization-dependent gain saturation in strained bulk semiconductor optical amplifiers. We assume that the polarized optical field can be decomposed into transverse electric and transverse magnetic components that have indirect interaction with each other via the gain saturation. The gain anisotropy due to tensile strain in the amplifier is accounted for by a population imbalance factor. The model is applied to a nonlinear polarization switch, for which results are obtained, that are in excellent agreement with experimental data. Finally, we describe an all-optical flip-flop memory that is based on two coupled nonlinear polarization switches.
\end{abstract}

Index Terms-Nonlinear polarization rotation, optical flip-flop memories, optical signal processing, optical switching, semiconductor optical amplifiers.

\section{INTRODUCTION}

$\mathbf{T}$ HE USE of polarization switches based on nonlinear polarization rotation in semiconductor optical amplifiers (SOAs) in optical signal processing applications is receiving considerable interest by many research groups. Applications of polarization switches to wavelength conversion are presented in [1]-[4]. Applications of polarization switches to optical time domain demultiplexing are presented in [5], [6]. Recently, the importance of polarization switches for all-optical logic has also been recognized [7]-[9]. Despite the large amount of experimental results that are published on polarization switches, the underlying concepts are not well understood [8]. In [10], theoretical results are published on polarization-dependent gain in SOAs in the context of optical switching and optical bistability, but their results are based on a microscopic model that is impractical as a design tool for optical switching configurations. We present a simple rate-equation model that can be used to model the switching characteristics of a polarization switch.

Our model is based on the decomposition of the polarized optical field into a transverse electric (TE) and transverse magnetic (TM) component. These modes propagate "independently" through the SOA, although they have indirect

Manuscript received June 25, 2002; revised August 29, 2002. This work was supported by the Netherlands Organization for Scientific Research (NWO) through the "NRC photonics" Grant.

H. J. S. Dorren, Y. Liu, M. T. Hill, and G.-D. Khoe are with the Cobra Research Institute, Eindhoven University of Technology, 5600 MB Eindhoven, The Netherlands.

D. Lenstra is with the Cobra Research Institute, Eindhoven University of Technology, 5600 MB Eindhoven, The Netherlands, and also with the Vrije Universiteit, FEW, Division of physics and astronomy, De Boelelaan 1081, 1081 HV Amsterdam, The Netherlands.

Digital Object Identifier 10.1109/JQE.2002.806200 interaction with each other via the gain saturation. We have accounted for different TE and TM gains by assuming that these polarizations couple to different hole reservoirs. This assumption is justified by the fact that the optical transitions occur between the $j=1 / 2$ type conduction band states and the $j=3 / 2$ type valence band states, where the latter correspond to the light-hole and heavy-hole valence bands. Two out of the three possible transition types are selected by the TE and TM polarizations, which define two corresponding inversions. In the isotropic bulk situation, these two transitions occur in a fully symmetric manner, but we are now interested in the case where tensile strain is built into the bulk medium, causing an asymmetry between the two transition types, such that TM will be favored over TE transitions. This will be modeled by introducing a population imbalance factor $f$.

Our rate equation model is applied to describe the switching characteristics of a polarization switch that is based on these principles. We find excellent agreement between our model and experimental data. Our results also reveal that there is an interesting similarity between the switching properties of a polarization switch and those of a Mach-Zehnder switch, which indicates that the polarization switch acts as a Mach-Zehnder switch, where the roles of the different light path are now played by the independently operating TE and TM modes of the optical field. Finally, we demonstrate an all-optical flip-flop memory that is made from two coupled polarization switches. This all-optical flip-flop has a simple structure, separate set and reset inputs and a large input wavelength range. We demonstrate the feasibility of the concept and we show that the contrast ratio between output states of flip-flop is over $20 \mathrm{~dB}$, while the switching power is less than $-3 \mathrm{dBm}$.

In Section II, we present the rate equation model that is based on the assumptions given above. Experimental results show that this model can explain the polarization-dependent gain saturation of a SOA. In Section III, we point out how our results can explain polarization switching in systems employing SOAs. We find excellent agreement between our model and experimental results. In Section IV, we demonstrate an optical flip-flop memory that is made from two coupled polarization switches. The paper is concluded with a discussion.

\section{MODEL}

We decompose the incoming arbitrarily polarized electric field in a component parallel to the layers in the waveguide 
TABLE I

SOA PARAMETER DEFINITIONS AND THEIR VALUES

\begin{tabular}{llll}
\hline Parameter & Symbol & Value & Units \\
\hline Confinement factor & $\Gamma^{T E}, \Gamma^{T M}$ & $0.2,0.14$ & \\
Phase modulation coefficients & $\alpha^{T E}, \alpha^{T M}$ & 5,5 & \\
Modal loss & $\alpha_{\text {int }}^{T E}, \alpha_{\text {int }}^{T M}$ & $0.27,0.27$ & $\mathrm{ps}^{-1}$ \\
Gain coefficient & $\xi^{T E}, \xi^{T M}$ & $7.0,6.5$ & $10^{-9} \mathrm{ps}^{-1}$ \\
Hole population imbalance factor & $f$ & 0.5 & \\
Electron-hole recombination time & $T_{1}$ & 500 & $\mathrm{ps}$ \\
Hole-hole relaxation time & $T_{2}$ & 0.1 & $\mathrm{ps}$ \\
Optical transition state number & $N_{0}$ & $10^{8}$ & \\
Group velocity & $v_{g}$ & 100 & $\mu \mathrm{m} / \mathrm{ps}$ \\
Electrical current & $I$ & 160 & $\mathrm{~mA}$ \\
SOA length & $L$ & 800 & $\mu \mathrm{m}$ \\
\hline
\end{tabular}

(TE mode) and a perpendicular component (TM mode). These two polarization directions are along the principal axes that diagonalize the wave propagation in the SOA. In fact, apart from their indirect interaction through the carrier dynamics in the device, these two polarizations propagate independently from each other. It is our aim to develop a model capable of describing the polarization behavior up to speeds of a few tens of gigahertz. In this case, we can use relatively simple propagation and rate equations, i.e., without necessity of taking into account the ultrafast (subpicosecond timescale) intraband relaxation dynamics.

The propagation equation for the TE-polarized electric field component $A^{\mathrm{TE}}(z, t)$ is given by

$$
\begin{aligned}
\left(\frac{\partial}{\partial t}+v_{g}^{\mathrm{TE}} \frac{\partial}{\partial z}\right) A^{\mathrm{TE}}(z, t)=\frac{1}{2} \Gamma^{\mathrm{TE}}\left(1+i \alpha^{\mathrm{TE}}\right) & \\
& \cdot g^{\mathrm{TE}}(z, t) A^{\mathrm{TE}}(z, t)-\frac{1}{2} \alpha_{\mathrm{int}}^{\mathrm{TE}} A^{\mathrm{TE}}(z, t) .
\end{aligned}
$$

Here, $A^{\mathrm{TE}}(z, t)$ is the weakly time and space-dependent complex envelope of the optical field, $v_{g}^{\mathrm{TE}}$ is the corresponding group velocity taken at the central frequency of the wave, $\Gamma^{\mathrm{TE}}$ is the confinement factor, $g^{\mathrm{TE}}(z, t)$ is the (real) gain function, $\alpha^{\mathrm{TE}}$ is the phase-modulation parameter, and $\alpha_{\mathrm{int}}^{\mathrm{TE}}$ is the modal loss. A similar rate equation holds for the complex field envelope $A^{\mathrm{TM}}(z, t)$ corresponding to the TM mode

$$
\begin{aligned}
\left(\frac{\partial}{\partial t}+v_{g}^{\mathrm{TM}}\right. & \left.\frac{\partial}{\partial z}\right) A^{\mathrm{TM}}(z, t)=\frac{1}{2} \Gamma^{\mathrm{TM}}\left(1+i \alpha^{\mathrm{TM}}\right) \\
& \cdot g^{\mathrm{TM}}(z, t) A^{\mathrm{TM}}(z, t)-\frac{1}{2} \alpha_{\mathrm{int}}^{\mathrm{TM}} A^{\mathrm{TM}}(z, t)
\end{aligned}
$$

where $A^{\mathrm{TM}}(z, t)$ is the weakly time- and space-dependent complex envelope of the optical field, $v_{g}^{\mathrm{TM}}$ is the corresponding group velocity, $\Gamma^{\mathrm{TM}}$ is the confinement factor, $g^{\mathrm{TM}}(z, t)$ the (real) gain function, $\alpha^{\mathrm{TM}}$ is the phase-modulation parameter,

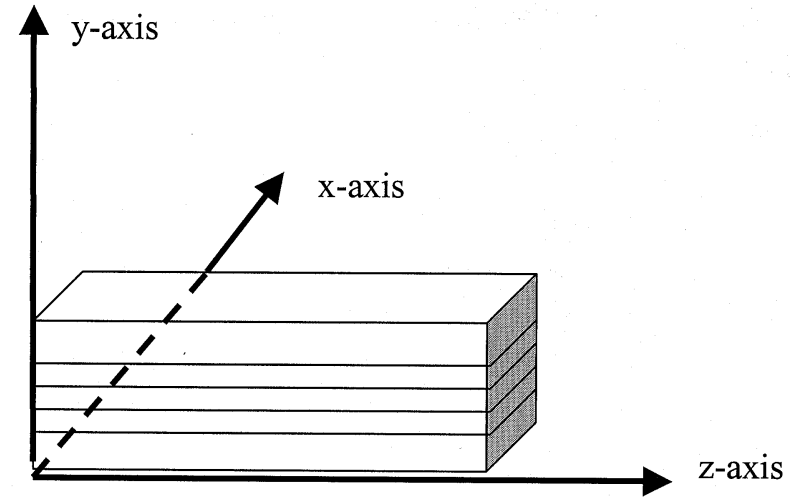

Fig. 1. Waveguide structure and definition of coordinate frame.

and $\alpha_{\mathrm{int}}^{\mathrm{TM}}$ is the modal loss. The envelopes for each polarization can be expressed as

$$
\begin{aligned}
A^{\mathrm{TE}}(z, t) & =\sqrt{S^{\mathrm{TE}}(z, t)} e^{i \phi^{\mathrm{TE}}(z, t)} \\
A^{\mathrm{TM}}(z, t) & =\sqrt{S^{\mathrm{TM}}(z, t)} e^{i \phi^{\mathrm{TM}}(z, t)}
\end{aligned}
$$

where $S^{\mathrm{TE}}(z, t)$ and $S^{\mathrm{TM}}(z, t)$ are the photon numbers and $\phi^{\mathrm{TE}}(z, t)$ and $\phi^{\mathrm{TM}}(z, t)$ are the phases for the TE and TM components.

The two optical modes have indirect interaction via the carriers. We assume that the TE and TM polarizations couple the electrons in the conduction band with two distinct reservoirs of holes. This assumption is justified by the fact that in zincblende structures such as GaAs and InP, optical transitions occur between $j=1 / 2$-type conduction band states and the $j=3 / 2$-type valence band states, where the latter are subdivided into light- and heavy-hole band states. In principle, three types of transitions can occur, but for the waveguide structure shown in Fig. 1, with the optical field propagating in the $z$ direction, the transitions with $z$ polarization are not activated. The remaining two transitions with $x$ and $y$ polarizations correspond to TE and TM polarizations, respectively. Hence, two out of the three possible transition types are selected by the TE and TM polarizations and these transitions define two relevant hole reservoirs, with corresponding numbers $n_{x}$ and $n_{y}$, respectively. In the isotropic bulk situation, the two transitions will occur in a fully symmetric manner, but we are now interested in the case where tensile strain is built into the bulk medium, causing an asymmetry between the two transition types, such that TM will be favored over TE transitions [17]. This compensates for extra TM waveguide losses and confinement factor differences so as to make the SOA polarization independent.

The number of electrons in the conduction band is denoted by $n_{c}(z, t)$, while the number of holes involved in the $x$ and $y$ transitions is denoted by $n_{x}(z, t)$ and $n_{y}(z, t)$. A more in-depth analysis shows that $n_{y}$ is just the number of holes in the light-hole band, while $n_{x}$ is made up of a mixture of light $(25 \%)$ and heavy $(75 \%)$ holes. In fact, due to tensile strain the light-hole population can be enhanced over the heavy holes, leading to enhanced TM transitions. This latter effect will be 


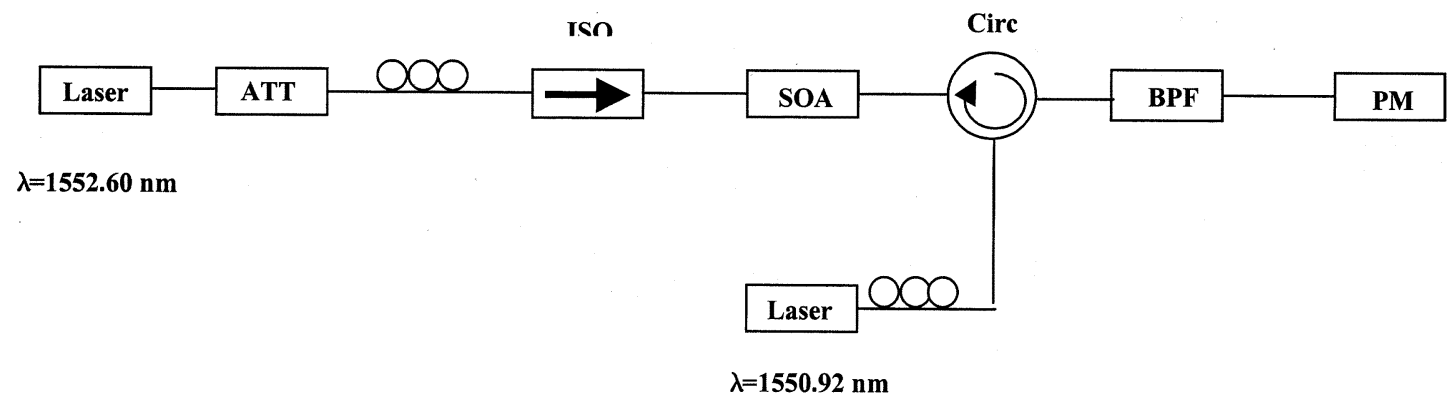

Fig. 2. Experimental setup that is used to measure the polarization-dependent SOA gain. SOA: Semiconductor optical amplifier. ISO: Isolator. ATT: Attenuator. Circ: Circulator. BPF: Bandpass filter. PM: Power meter.

accounted for by an imbalance factor $f$ [see (8)-(10)]. The (linearized) gain $g^{\mathrm{TE}}(z, t)$ for $\mathrm{TE}$ polarization is given by

$$
g^{\mathrm{TE}}(z, t)=\xi^{\mathrm{TE}}\left[n_{c}(z, t)+n_{x}(z, t)-N_{0}\right]
$$

where $\xi^{\mathrm{TE}}$ is the gain coefficient for the TE mode and $N_{0}$ is the total number of electronic states involved in the optical transition. Similarly, the gain $g^{\mathrm{TM}}(z, t)$ can be expressed as

$$
g^{\mathrm{TM}}(z, t)=\xi^{\mathrm{TM}}\left[n_{c}(z, t)+n_{y}(z, t)-N_{0}\right]
$$

where $\xi^{\mathrm{TM}}$ is the gain coefficient for the TM mode. In cases of high-intensity optical beams, one should correct $\xi^{\mathrm{TE} / \mathrm{TM}}$ for saturation due to the carrier heating according to

$$
\xi^{\mathrm{TE} / \mathrm{TM}}=\frac{\xi_{0}^{\mathrm{TE} / \mathrm{TM}}}{1+\varepsilon S^{\mathrm{TE} / \mathrm{TM}}}
$$

where $\varepsilon$ is typically $10^{-7}$ per photon present in the SOA [11]. In the experiments that follow, we use optical fields that have a much lower intensity so that in good approximation $\xi^{\mathrm{TE} / \mathrm{TM}}=$ $\xi_{0}^{\mathrm{TE} / \mathrm{TM}}$. In writing down (4) and (5), we tacitly assumed that the semiconducting medium in the active layer gives rise to anisotropic gain, such as can be realized in a bulk layer with tensile strain [12]. If we assume that the total number of holes is equal to the number of electrons

$$
n_{c}(z, t)=n_{x}(z, t)+n_{y}(z, t)
$$

and substitute this into (5) and (6), we can express $g^{\mathrm{TE}}(z, t)$ and $g^{\mathrm{TM}}(z, t)$ as

$$
\begin{aligned}
g^{\mathrm{TE}}(z, t) & =\xi^{\mathrm{TE}}\left[2 n_{x}(z, t)+n_{y}(z, t)-N_{0}\right] \\
g^{\mathrm{TM}}(z, t) & =\xi^{\mathrm{TM}}\left[2 n_{y}(z, t)+n_{x}(z, t)-N_{0}\right] .
\end{aligned}
$$

The rate-equation for $n_{x}(z, t)$ can be written as

$$
\frac{\partial n_{x}(z, t)}{\partial t}=-\frac{n_{x}(z, t)-\bar{n}_{x}}{T_{1}}-\frac{n_{x}(z, t)-f n_{y}(z, t)}{T_{2}}
$$

and similarly for $n_{y}(z, t)$

$$
\begin{aligned}
& \frac{\partial n_{y}(z, t)}{\partial t}=-\frac{n_{y}(z, t)-\bar{n}_{y}}{T_{1}}-\frac{f n_{y}(z, t)-n_{x}(z, t)}{T_{2}} \\
& -g^{\mathrm{TM}}(z, t) S^{\mathrm{TM}}(z, t)
\end{aligned}
$$

where $\bar{n}_{x}$ and $\bar{n}_{y}$ are the respective equilibrium values determined by the applied pump current as will be discussed below, $T_{1}$ is the electron-hole recombination time, and $T_{2}$ is the inter-hole relaxation time. The last terms in the right-hand sides of (8) and (9) account for the stimulated recombinations. It should be noted that the inter-hole relaxation time $T_{2}(\sim 100 \mathrm{fs})$ is much shorter than the electron hole recombination time $T_{1}$ ( $\sim 500$ ps). Since we do not consider applications that involve ultrafast dynamics here, the two populations $n_{x}$ and $n_{y}$ will be clamped tightly together, i.e.,

$$
n_{x}(z, t)=f n_{y}(z, t)
$$

In case of unstrained bulk material, the gain will be isotropic and $f=1$. In case of tensile strain, TM gain will be larger than TE, i.e., $f<1$. For the equilibrium values, consistent with (10), we can write

$$
\bar{n}_{x}=\frac{\bar{n} f}{1+f}, \quad \bar{n}_{y}=\frac{\bar{n}}{1+f}
$$

where

$$
\bar{n}=\frac{I}{e} T_{1}
$$

and $I$ is the electric current and $e$ is the electric unit charge. In (8)-(11), $f$ expresses the magnitude of the anisotropy.

Equations (1)-(12) form a closed set of equations. First, we will calculate the small-signal gain. To this end, we substitute the equilibrium values (11) in the gain expressions (7) and obtain the small-signal gain $g_{0}^{\mathrm{TE} / \mathrm{TM}}$

$$
\begin{aligned}
& g_{0}^{\mathrm{TE}}=\xi^{\mathrm{TE}}\left[\left(\frac{1+2 f}{1+f}\right) \bar{n}-N_{0}\right] \\
& g_{0}^{\mathrm{TM}}=\xi^{\mathrm{TM}}\left[\left(\frac{2+f}{1+f}\right) \bar{n}-N_{0}\right] .
\end{aligned}
$$

These expressions are quite general, but derived under the assumption of linear relationship between gain and carrier numbers. This implies that (13) can only be used in a small interval of the pump current. Within this given interval the parameter values occurring in (13) can be determined, but for different intervals, different parameter values will be obtained.

According to (1) and (2), the net amplifications by the SOA (in decibels), in absence of spatial inhomogeneity, are given by

$$
4.343 \times\left(\Gamma^{\mathrm{TE}} g^{\mathrm{TE}}-\alpha_{\mathrm{int}}^{\mathrm{TE}}\right) \frac{L}{v_{g}^{\mathrm{TE}}}, \quad \text { for } \mathrm{TE}
$$

and

$$
4.343 \times\left(\Gamma^{\mathrm{TM}} g^{\mathrm{TM}}-\alpha_{\mathrm{int}}^{\mathrm{TM}}\right) \frac{L}{v_{g}^{\mathrm{TM}}}, \quad \text { for } \mathrm{TM}
$$




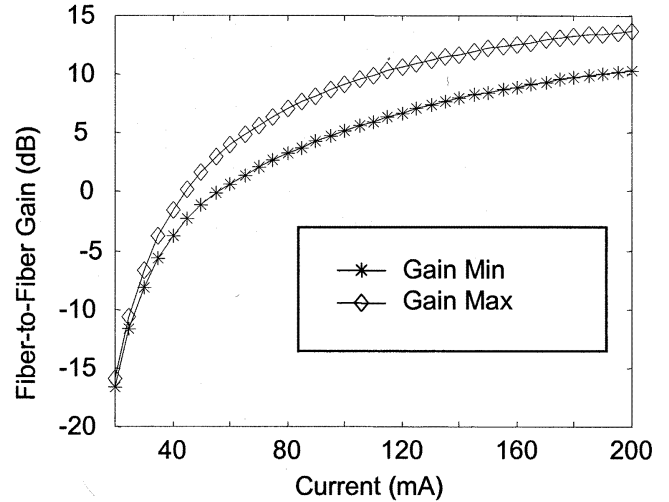

(a)

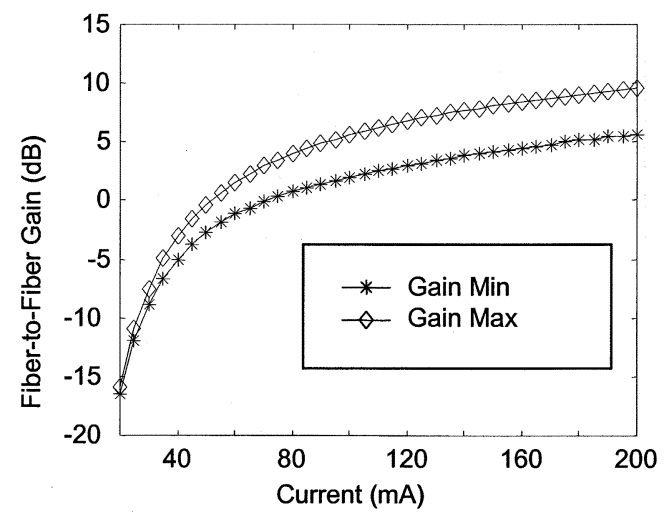

(b)

Fig. 3. (a) Measured polarization-dependent small-signal gain for the TE mode and TM mode as a function of the injected current $I$. (b) Similar to (a), but now with a saturating control signal. The intensity of the control signal is $0.32 \mathrm{dBm}$.

where $L$ is the length of the SOA. The small-signal amplification can be obtained by replacing $g^{\mathrm{TE}}$ by $g_{0}^{\mathrm{TE}}$ and $g^{\mathrm{TM}}$ by $g_{0}^{\mathrm{TM}}$ in (14) and (15).

The experimental setup that is used to measure the polarization-dependent gain of the SOA is shown in Fig. 2. A laser source emits a continuous wave $(\mathrm{CW})$ probe beam at a wavelength of $1552.60 \mathrm{~nm}$. This probe beam is fed into the SOA via an attenuator and an isolator. The SOA was manufactured by JDS-Uniphase and employs a strained-bulk active region with a length of $800 \mu \mathrm{m}$. An attenuator is used to assure the light that enters the SOA has a low intensity $(-15.24 \mathrm{dBm})$ so that the SOA is operated in the linear regime (with this, we mean that no gain saturation is introduced by the probe beam). The SOA can be saturated by a high-intensity CW-pump beam at a wavelength of $1550.90 \mathrm{~nm}$ that enters from the opposite direction. The SOA output is fed into a power meter via a circulator. A tuneable bandpass filter with a bandwidth of $2 \mathrm{~nm}$ is used to filter out the spontaneous emission that is produced by the SOA. The polarization controllers are used to adjust the polarization of the input signals (pump and probe) to the orientation of the SOA layers.

As a first experiment, the polarization-dependent gain is measured as a function of the injected current $I$ in the absence of a saturating control beam. This is done by adjusting the polarization of the small-signal probe beam in such a way that the minimum and maximum amplification is measured. The results are

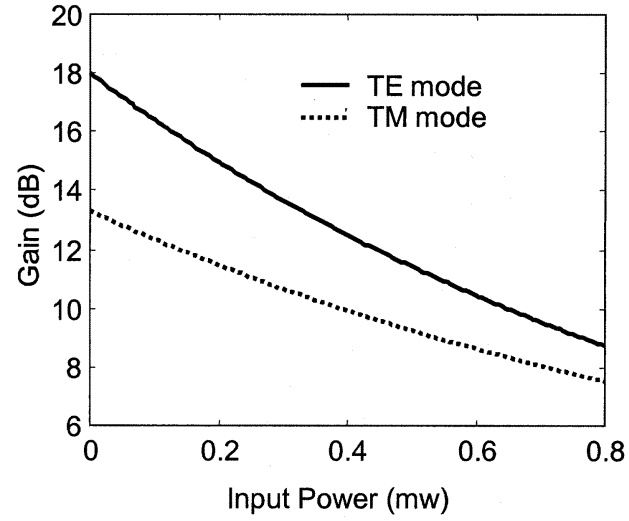

Fig. 4. Computed SOA gain for the TE mode (solid line) and the TM mode (dashed line) as a function of the intensity of a saturating control signal. The SOA was pumped with $160 \mathrm{~mA}$

presented in Fig. 3(a), where the curve with the maximum amplification is attributed to the TE mode and the curve with minimum amplification is attributed to the TM mode. If we compensate for the coupling losses, estimated to be $5.6 \mathrm{~dB}$ (this includes two times $2.5-\mathrm{dB}$ facet losses and two times $0.3-\mathrm{dB}$ connector losses), it follows from Fig. 3(a) that for a current $I=160$ $\mathrm{mA}$, the gain of the TE mode equals to $18.1 \mathrm{~dB}$ and that of the TM mode $13.6 \mathrm{~dB}$. Moreover, if we use the following parameter values: $\alpha_{\mathrm{int}}^{\mathrm{TE}}=\alpha_{\mathrm{int}}^{\mathrm{TM}}=0.27 \mathrm{ps}^{-1}, f=0.5, N_{0}=10^{8}$, $T_{1}=500 \mathrm{ps}, e=1.610^{-19} \mathrm{C}, v_{g}^{\mathrm{TE}}=v_{g}^{\mathrm{TM}}=100 \mu \mathrm{m} / \mathrm{ps}$, $\Gamma^{\mathrm{TE}}=0.2$, and $\Gamma^{\mathrm{TM}}=0.14$ [13], [14], then it follows from (14) and (15) that $\xi^{\mathrm{TE}}=7.010^{-9} \mathrm{ps}^{-1}$ and $\xi^{\mathrm{TM}}=6.510^{-9}$ $\mathrm{ps}^{-1}$.

If a saturating control beam is also injected into the SOA, the gain can be computed by using (7)-(9), (14), and (15). The optical power $P^{\mathrm{TE} / \mathrm{TM}}$ for each mode is related to the intensity $S^{\mathrm{TE} / \mathrm{TM}}$ according to

$$
S^{\mathrm{TE} / \mathrm{TM}}=\frac{P^{\mathrm{TE} / \mathrm{TM}}}{\hbar \omega} \frac{L}{v_{g}^{\mathrm{TE} / \mathrm{TM}}}
$$

where $\hbar$ is Planck's constant and $\omega$ is the optical frequency. In Fig. 3(b), the saturated gain is plotted as a function of the injected current if a saturating pump signal with an intensity of $0.32 \mathrm{dBm}$ is applied. The polarization of the pump beam was adjusted in such a way that maximum gain saturation for both the TE and TM modes was obtained. In Fig. 4, the computed gain saturation is presented for the two modes as a function of the injected optical power at a bias current of $160 \mathrm{~mA}$, while it is assumed that $50 \%$ of the injected power is used to saturate the TE mode and the other $50 \%$ of the injected power is used to saturate the TM mode. If we correct for $2.8-\mathrm{dB}$ coupling losses of the pump beam, and if we assume $\hbar \omega=0.8 \mathrm{eV}$, it follows from (16) that under these circumstances, a pump beam with an intensity of $0.32 \mathrm{dBm}$ leads to a saturated gain of $13.4 \mathrm{~dB}$ for the TE mode and 10.5 for the TM mode. This is in agreement with the experimental data that are presented in Fig. 3(b).

We have also compared the experimental gain saturation and the computed gain saturation for other intensities of the saturating control beam, and we find that the experimental results are in good agreement with the theoretical results. It should be remarked, however, that from an experimental point of view, it 


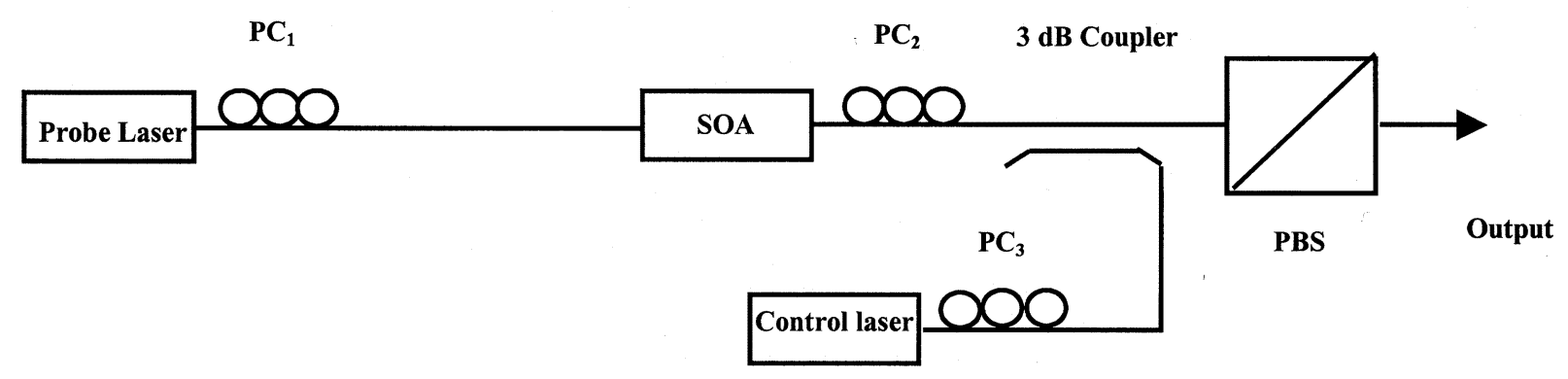

Fig. 5. Schematic setup of a nonlinear polarization switch. PC: polarization controller. SOA: semiconductor optical amplifier. PBS: polarizing beam splitter.

is difficult to control the intensities of the light that is injected in each mode.

We have shown that our SOA model can accurately describe the polarization-dependent gain saturation. It should be noted, however, that the SOA parameters $N_{0}, T_{1}, \Gamma^{\mathrm{TE} / \mathrm{TM}}, \alpha_{\mathrm{int}}^{\mathrm{TE} / \mathrm{TM}}$, and $v_{g}^{\mathrm{TE} / \mathrm{TM}}$ cannot be estimated accurately. We solve this problem by compensating the combined uncertainties in these parameter values by assigning values to $f$ and $\xi^{\mathrm{TE} / \mathrm{TM}}$ in such a way that the (measured) fiber-to-fiber gain is reproduced. In the most simple approach, one would choose $\xi^{\mathrm{TE}}=\xi^{\mathrm{TM}}$, as this would be exact in the case of isotropic gain, so that $f$ can be estimated from the measured TE and TM gain curves by using (14) and (15). In this case, the polarization-dependent gain would be totally explained by the band-filling effects that are represented by the factor $f$. However, this leads to gain saturation that is not in agreement with experimental data. We have, therefore, chosen to allow for a small difference in the values for $\xi^{\mathrm{TE}}$ and $\xi^{\mathrm{TM}}$ (as this could be due to a small difference in effective transition strength). This approach leads to a gain saturation that is in good agreement with experimental data. The difficulties in estimating $f$ and $\xi^{\mathrm{TE} / \mathrm{TM}}$ may be inherent to our modeling the SOA strain in terms of a population imbalance factor $f$. In a more accurate-but also much more complicated-model, one can calculate the band structure and transition matrix elements in the presence of tensile strain and keep track of the different optical transitions involved as well as the relevant populations. This would, however, extend beyond the scope of the present approach.

\section{NONLINEAR POLARIZATION SWITCHING}

The principle of polarization-dependent SOA gain discussed in the previous section will now be applied to a nonlinear polarization switch (PSW) schematically indicated in Fig. 5. The PSW is made from two laser sources: a SOA, three polarization controllers, and a polarization beam splitter (PBS) [5]. The first laser (Laser 1) emits a CW probe beam at wavelength $\lambda_{1}$ that is fed into the SOA. The SOA output is sent into the PBS. One polarization controller $\left(\mathrm{PC}_{1}\right)$ is used to adjust the polarization direction of the input signal at approximately $45^{\circ}$ to the orientation of the SOA layers, while the second polarization controller $\left(\mathrm{PC}_{2}\right)$ adjusts the polarization of the (amplified) output light to the orientation of the PBS. The SOA gain can be saturated by injection of a high-intensity pump (control) signal produced by the second laser (Laser 2). The wavelength $\lambda_{2}$ of the pump beam should be distinguishable from the wavelength of

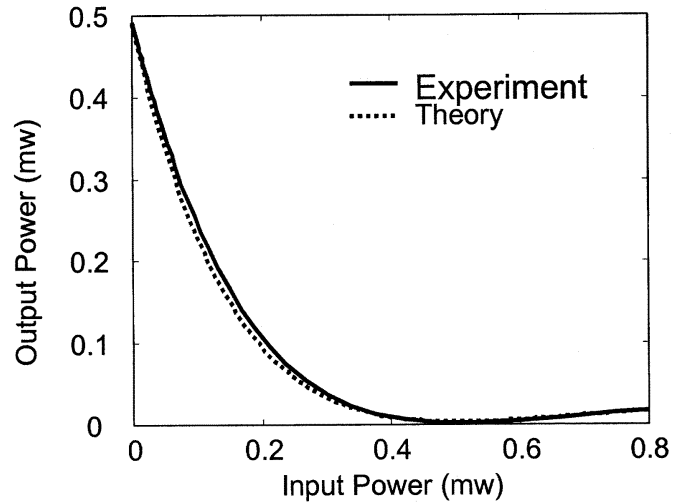

Fig. 6. Output of the setup of Fig. 5. The system is tuned in such a way that the maximum output intensity of the polarization switch is equal to the intensity of the control light that is required to suppress the output of the polarization switch. Horizontal axis: represents the switching intensity. Vertical axis: represents the output intensity of the polarization switch. Solid curve: experimental result. Dashed curve: theoretical result.

the probe beam if the two beams co-propagate. If the control light counter-propagates with the probe signal, the two wavelengths may be identical. The polarization of the light from Laser 2 is controlled by the third polarization controller $\left(\mathrm{PC}_{3}\right)$.

The solid curve in Fig. 6 shows the experimental PBS output as a function of the intensity of the saturating control light. The SOA used in this experiment was pumped with $162 \mathrm{~mA}$ of current. Fig. 6 clearly demonstrates that a control beam of sufficient intensity suppresses the output of the PSW. This effect is a consequence of the polarization-dependent gain saturation described in Section II. The gain saturation generally leads to different refractive-index changes for TE and TM, which results in a saturation-induced phase difference between these modes. If this phase difference is an odd multiple of $\pi$, the output from the PBS is suppressed, i.e., switched off.

In the experiment, the polarization direction of the input probe light is approximately at $45^{\circ}$ to the layers of the SOA, but not exactly. This is because our SOA had a polarization sensitivity of almost $4 \mathrm{~dB}$ at $1550 \mathrm{~nm}$, implying a difference in the saturation properties of TE and TM modes also. The input angle is carefully adjusted to compensate for this, thus achieving more than $20-\mathrm{dB}$ switching contrast ratio.

The phase difference $\theta$ between the TE and TM modes can be computed from (1) and (2)

$$
\theta=\phi^{\mathrm{TE}}-\phi^{\mathrm{TM}}=\frac{1}{2}\left(\frac{\alpha^{\mathrm{TE}} \Gamma^{\mathrm{TE}} g^{\mathrm{TE}}}{v_{g}^{\mathrm{TE}}}-\frac{\alpha^{\mathrm{TM}} \Gamma^{\mathrm{TM}} g^{\mathrm{TM}}}{v_{g}^{\mathrm{TM}}}\right) L
$$




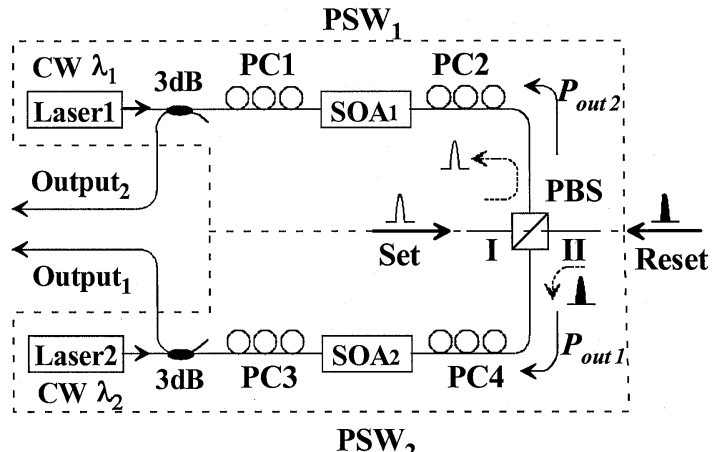

Fig. 7. Configuration of the all-optical flip-flop based on two polarization switches. CW: Continuous wave. PSW: Polarization switch. PC: Polarization controller. SOA: Semiconductor optical amplifier. PBS: Polarization beam splitter. Mux: Optical multiplexer. $\mathrm{P}_{\text {out } 1}$ : the output of $\mathrm{PSW}_{1}, \mathrm{P}_{\text {out } 2}$ : the output of $\mathrm{PSW}_{2}$.

where spatial inhomogeneity is neglected altogether. Such effects can be taken into account, but then the propagation equation should be numerically integrated. In the cases considered here, (17) turns out to be an adequate approximation. The intensity $S_{\text {out }}$ of the light that outputs the PBS is given by

$$
S_{\text {out }}=S^{\mathrm{TE}}+S^{\mathrm{TM}}+2 \sqrt{S^{\mathrm{TE}} S^{\mathrm{TM}}} \cos \theta
$$

where

$$
\begin{aligned}
S^{\mathrm{TE}} & =S_{\mathrm{in}}^{\mathrm{TE}} e^{\left(\Gamma^{\mathrm{TE}} g^{\mathrm{TE}}-\alpha_{\mathrm{int}}^{\mathrm{TE}}\right)\left(L / v_{g}^{\mathrm{TE}}\right)} \\
S^{\mathrm{TM}} & =S_{\mathrm{in}}^{\mathrm{TM}} e^{\left(\Gamma^{\mathrm{TM}} g^{\mathrm{TM}}-\alpha_{\mathrm{int}}^{\mathrm{TM}}\right)\left(L / v_{g}^{\mathrm{TM}}\right)}
\end{aligned}
$$

and $S_{\mathrm{in}}^{\mathrm{TE} / \mathrm{TM}}$ in (19) represent the intensities of the TE and TM components of the probe beam at the SOA input. The gains $g^{\mathrm{TE} / \mathrm{TM}}$ can be computed from (7) and (9). Once the gain for each mode is known, the phase difference can be computed by using (17).

If we use the SOA parameters that are presented in Section II, we can compute $S_{\text {out }}$ as a function of the intensity of a control beam by using (17)-(19). The result is shown by the dashed curve in Fig. 6 if $\alpha_{\mathrm{TE}}=\alpha_{\mathrm{TM}}=5$. It is clearly visible in Fig. 6 that our SOA model leads to results that are in excellent agreement with the experimental data. Note that curves in Fig. 6 are similar to the ones presented in [15], in which the suppressed output of an active Mach-Zehnder interferometer is discussed. This reflects that the nonlinear polarization switch operates in a similar fashion as a Mach-Zehnder interferometer switch, since in the PSW, the role of the different light paths is played by the different polarizations.

\section{All-Optical Flip-Flop BASEd on NONLINEAR PSWs}

Similar to [15], an optical flip-flop can be realized by coupling two identical PSWs as shown in Fig. 7. The first PSW, hereafter to be called PSW 1 , outputs light that is injected into the second polarization switch (to be called $\mathrm{PSW}_{2}$ ) via the PBS. Hence, the output light from $\mathrm{PSW}_{1}$ acts as a saturating control signal that can suppress $\mathrm{PSW}_{2}$ and vice versa. The solid curve in Fig. 8 represents the intensity $\mathrm{P}_{\text {out } 1}$ of the light that outputs $\mathrm{PSW}_{1}$ as a function of the intensity $\mathrm{P}_{\text {out } 2}$ of the light that outputs $\mathrm{PSW}_{2}$ and saturates the SOA in $\mathrm{PSW}_{1}$. Conversely, the dashed curve in Fig. 8 represents the intensity $\mathrm{P}_{\text {out } 2}$ of the light

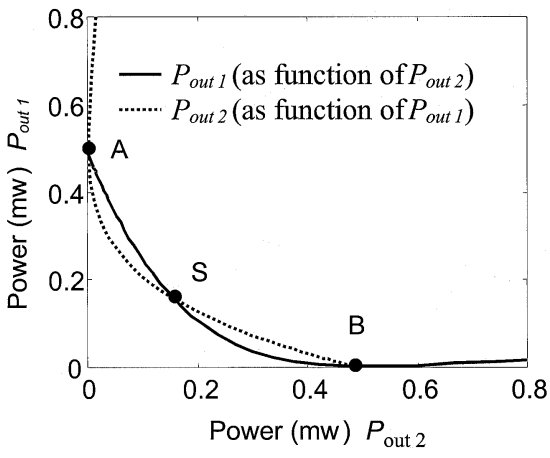

Fig. 8. Intensity $\mathrm{P}_{\text {out } 1}$ of the light that outputs $\mathrm{PSW}_{1}$ as a function of the intensity $\mathrm{P}_{\text {out } 2}$ of the light that outputs $\mathrm{PSW}_{2}$ (solid curve). Intensity $\mathrm{P}_{\text {out }} 2$ of the light that outputs $\mathrm{PSW}_{2}$ as a function of the intensity $\mathrm{P}_{\text {out } 1}$ of the light that outputs PSW $_{1}$ (dashed curve). It is visible that the two curves are complementary. Both curves are experimental results.

that outputs $\mathrm{PSW}_{2}$ as a function of the amount of light $\mathrm{P}_{\text {out } 1}$ that outputs $\mathrm{PSW}_{1}$ and saturates the SOA in $\mathrm{PSW}_{2}$. Both curves in Fig. 8 are experimental results. They are obtained under the conditions that are presented later in this section. Since the polarization switches are identical, the solid curve is complementary to the dashed curve. At point $\mathrm{A}, \mathrm{PSW}_{2}$ is suppressed and $\mathrm{PSW}_{1}$ is dominant while at Point $\mathrm{B}, \mathrm{PSW}_{1}$ is suppressed and $\mathrm{PSW}_{2}$ is dominant. Point $\mathrm{S}$ is an unstable point [16].

The system of two coupled PSWs can function as an optical flip-flop as follows. The state of the flip-flop can be determined by observing the amount of light at the outputs of the PSW. In State $1, \mathrm{PSW}_{1}$ dominates and suppresses $\mathrm{PSW}_{2}$, while in State 2, $\mathrm{PSW}_{2}$ dominates and suppresses $\mathrm{PSW}_{1}$. To switch the flip-flop between the states, light can be injected in the PSW that dominates (that is the one injecting the most light into the other PSW) via the set state and reset ports. The injected light reduces the light exiting the dominant PSW, which allows the suppressed PSW to increase its light output and become the dominant switch.

The all-optical flip-flop is implemented as in Fig. 7. Lasers 1 and 2 emit CW light at wavelengths $\lambda_{1}=1549.32 \mathrm{~nm}$ and $\lambda_{2}=$ $1550.92 \mathrm{~nm}$, respectively. However, it is not essential to bias the PSWs with light at different wavelengths. The output power is $-3.34 \mathrm{dBm}$ for Laser 1 and $-3.05 \mathrm{dBm}$ for Laser 2. $\mathrm{SOA}_{1}$ is biased with $163.97 \mathrm{~mA}$ of current and $\mathrm{SOA}_{2}$ is biased with $161.86 \mathrm{~mA}$ of current. The PBS has four ports and an extinction ratio of $30 \mathrm{~dB}$. $\mathrm{PSW}_{1}$ and $\mathrm{PSW}_{2}$ are coupled to each other via the PBS.

The static operation of the flip-flop that is discussed in the previous section is presented in Fig. 8. The flip-flop operation is demonstrated by toggling the state of the flip-flop by injecting a regular sequence of optical pulses into the switch that was currently the master. These pulses had a wavelength of $1552.52 \mathrm{~nm}$ and duration of $150 \mathrm{~ns}$. The pulses were injected in the master once every $1.85 \mathrm{~ms}$ through the set and reset port (see Fig. 7). Fig. 9 shows the oscilloscope traces of the optical output power of flip-flop for each state. The optical peak power for the set and reset pulses were $-3.91 \mathrm{dBm}$ and $-4.35 \mathrm{dBm}$. In Fig. 9, regular toggling between flip-flop states every 1.85 microseconds can be observed. Furthermore, it is visible that the flip-flop state is stable in the time between the state changes. Also, the contrast 


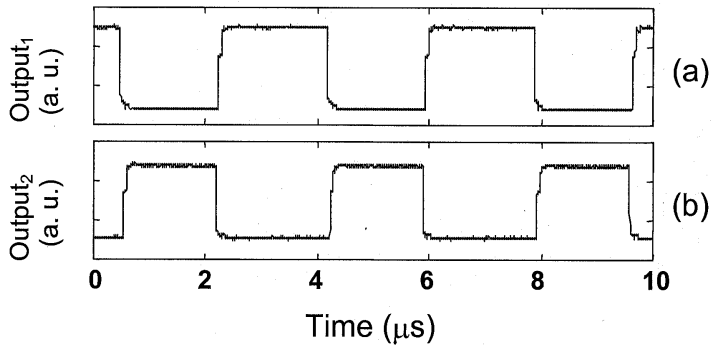

Fig. 9. Regular toggling between the two flip-flop states if, every $1.85 \mathrm{~ms}$, a set or reset pulse is injected.
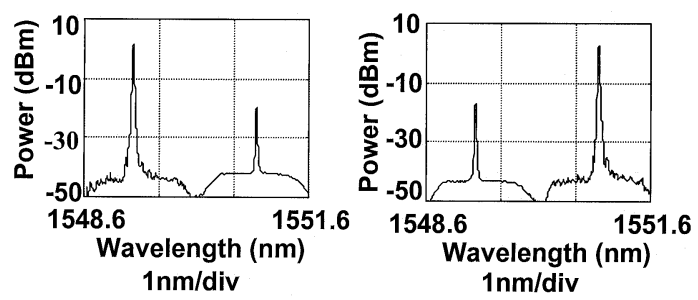

Fig. 10. Optical spectrum of the flip-flop output states. It can be observed that $20-\mathrm{dB}$ contrast between the states is obtained. On the left, the spectrum $\mathrm{PSW}_{1}$ dominates. On the right, the spectrum $\mathrm{PSW}_{2}$ dominates.

between States 1 and 2 was investigated by using a spectrum analyzer. The result is presented in Fig. 10. It turns out that the contrast ratio between the two states of the flip-flop is over $20 \mathrm{~dB}$.

\section{CONCLUSIONS}

We have presented a simple rate-equation model that is capable of describing the behavior of polarization switches based on nonlinear polarization rotation in strained bulk SOAs. This model is based on the assumption that TE and TM components of the light correspond to the two principle axes of the SOA while the carriers establish an indirect coupling between the components. The effect of tensile strain is accounted for by a population imbalance factor $f$ and a small difference in gain coefficients for TE and TM.

The polarization-dependent gain saturation of the SOA can be explained with our model. The TE and TM modes experience different refractive indexes, which leads to a phase difference between the two modes. The operation of a nonlinear PSW is based on this principle, in which the phase difference is used to suppress the output of the PSW. The nonlinear polarization switch turns out to behave similarly as a Mach-Zehnder interferometer, in which the roles of the different light paths are taken by the independently propagating TE and TM modes.

Finally, we present an all-optical flip-flop memory based on two coupled PSWs. The contrast ratio between output states of flip-flop is over $20 \mathrm{~dB}$ and the optical switching power is less than $-3 \mathrm{dBm}$. The speed of this flip-flop is determined by the speed of the PSW and the propagation distance between two SOAs. In the experimental setup, approximately $12 \mathrm{~m}$ of fiber is used between the two SOAs, which implies that about at least $100 \mathrm{~ns}$ are required for the states of the flip-flop to change. However, integrated versions of the flip-flop could reduce the distance two SOAs to several millimeters. In this case, the speed of flip-flop is dominated by the speed of the PSW. It has been demonstrated that the switches can operate at $10 \mathrm{GHz}$ [5]; thus, we expect the presented flip-flop to reach at least similar speeds.

\section{REFERENCES}

[1] M. F. C. Stephens, M. Asghari, R. V. Petty, and I. H. White, "Demonstration of ultrafast wavelength conversion utilizing birefringence in semiconductor optical amplifiers," IEEE Photon. Technol. Lett., vol. 9, pp. 449-451, 1997.

[2] M. Asghari, I. H. White, and R. Penty, "Wavelength conversion using semiconductor optical amplifiers," J. Lightwave Technol., vol. 15, pp. 1181-1190, 1997

[3] H. Soto, D. Erasme, and G. Guekos, "Cross-polarization modulation in semiconductor optical amplifiers," IEEE Photon. Technol. Lett., vol. 11, pp. 970-972, 1999.

[4] Y. Liu, M. T. Hill, E. Tangdiongga, H. de Waardt, N. Calabretta, G. D. Khoe, and H. J. S. Dorren, "All-optical wavelength converter based on cross-polarization modulation in a single semiconductor optical amplifier," Proc. ECOC 2002, to be published.

[5] M. F. C. Stephens, A. E. Kelly, D. Nesset, A. Wonfor, and R. V. Penty, "Demonstration of time demultiplexing from $40 \mathrm{~Gb} / \mathrm{s}$ to $10 \mathrm{~Gb} / \mathrm{s}$ via polarization switching in a semiconductor optical amplifier," in Proc. CLEO/Europe '98, 1998, pp. 383-384.

[6] D. M. Patrick, A. D. Ellis, D. A. O. Davies, M. C. Tatham, and G. Sherlock, "Demultiplexing using polarization rotation in a semiconductor laser amplifier," Electron. Lett., vol. 30, pp. 341-342, 1994.

[7] H. Soto, D. Erasme, and G. Guekos, "All-optical switch demonstration using a birefringence effect in a semiconductor optical amplifier," in Proc. CLEO/Pacific Rim '99, 1999, pp. 888-889.

[8] R. J. Manning, A. Antonopoulos, R. Le Roux, and A. E. Kelly, "Experimental measurement of nonlinear polarization rotation in semiconductor optical amplifiers," Electron. Lett., vol. 37, pp. 229-231, 2001.

[9] H. Soto, D. Erasme, and G. Guekos, "5-Gbit/s XOR optical gate based on cross-polarization modulation in semiconductor optical amplifiers," IEEE Photon. Technol. Lett., vol. 13, pp. 335-337, 2001.

[10] Y. Takahashi, A. Neogi, and H. Kawaguchi, "Polarization dependent nonlinear gain in semiconductor optical amplifiers," IEEE J. Quantum Electron., vol. 34, pp. 1660-1672, Sept. 1998.

[11] G. P. Agrawal and N. K. Dutta, Long-Wavelength Semiconductor Lasers. New York: Van Nostrand, 1986.

[12] T. Kakitsuka, Y. Shibata, M. Itoh, Y. Kadota, Y. Tohmori, and Y. Yoshikuni, "Influence of buried structure on polarization sensivity in strained bulk semiconductor optical amplifiers," IEEE J. Quantum Electron., vol. 38, pp. 85-91, Jan. 2002.

[13] T. D. Visser, H. Blok, and D. Lenstra, "Theory of polarization dependent amplification in a slab waveguide with anisotropic gain and losses," IEEE J. Quantum Electron., vol. 35, pp. 240-249, Feb. 1999.

[14] T. D. Visser, H. Blok, B. Demeulenaere, and D. Lenstra, "Confinement factors and gain in optical amplifiers," IEEE J. Quantum Electron., vol. 33, pp. 1763-1766, Oct. 1997.

[15] M. T. Hill, H. de Waardt, G. D. Khoe, and H. J. S. Dorren, "Fast optical flip-flop by use of Mach-Zehnder interferometers," Microwave Opt. Technol. Lett., vol. 31, pp. 411-415, 2001.

[16] — - "All-optical flip-flop based on coupled laser diodes," IEEE $J$. Quantum Electron., vol. 37, pp. 405-413, Mar. 2001.

[17] E. Kapon, Ed., Semiconductor Lasers 1. New York: Academic, 1999, sec. 2.3.

H. J. S. Dorren received the M.Sc. degree in theoretical physics in 1991 and the Ph.D. degree in 1995, both from Utrecht University, Utrecht, The Netherlands. After a postdoctoral position at Utrecht University, he accepted a postdoctoral position in Telecommunication Technology at Eindhoven University of Technology, Eindhoven, The Netherlands, in 1996. He was also with KPN Research (part-time), the largest network operator in the Netherlands. In both positions, he was involved in research on wavelength-division multiplexing network management. Since 1999, he has been an Assistant Professor at Eindhoven University, where his research includes optical signal processing, optical packet switching, and ultra-fast carrier dynamics in semiconductor materials. 


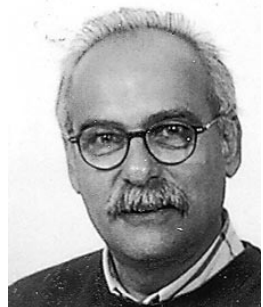

Daan Lenstra (M'97) was born in Amsterdam, The Netherlands, in 1947. He received the M.Sc. degree in theoretical physics from the University of Groningen, Groningen, The Netherlands, and the Ph.D. degree from Delft University of Technology, Delft, The Netherlands. His thesis work was on polarization effects in gas lasers.

Since 1979, he has researched topics in quantum electronics, laser physics, and condensed matter physics. In 1991, he joined the Vrije Universiteit, Amsterdam, The Netherlands, holding a Chair in Theoretical Quantum Electronics. Since September 2000, he has also been a Professor (part-time) with the Department of Electrical Engineering, Eindhoven University of Technology, Eindhoven, The Netherlands. His areas of research include nonlinear and ultra-fast dynamics of semiconductor optical amplifiers and diode lasers, quantum optics in small semiconductor structures, and near-field optics. He has (co)authored more than 170 publications in international scientific journals and (co)edited six books and special journal issues.

Yong Liu (S' 97) was born in Sichuan Province, China, in 1970. He received the Master's degree in electronic engineering from the University of Electronic Science and Technology of China in 1994. He is currently working woard the $\mathrm{Ph} . \mathrm{D}$. degree at Eindhoven University of Technology, Eindhoven, The Netherlands, where his field of research is all-optical buffering using all-optical signal processing.

From 1994 to 2000 , he was involved in teaching and research at the University of Electronic Science and Technology of China.

Martin T. Hill (S'96-M'97) was born in Sydney, Australia, in 1968. He received the B.E. degree (with first-class honors) in 1990 and the M.Eng.Sc. degree in 1992, both from the University of Western Australia, and the Ph.D. degree in 1997 from Curtin University of Technology, Perth, Western Australia.

During 1990, he was a member of Technical Staff at QPSX Communications Pty. Ltd. From 1993 to 1998, he was with the Australian Telecommunications Research Institute. Since 1998, he has been with the Department of Electrical Engineering, Technical University of Eindhoven, Eindhoven, The Netherlands, where he is involved in research on photonic components for optical packet switching.

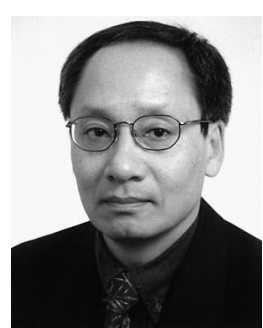

Giok-Djan Khoe (F'91) was born in Magelang, Indonesia, in 1946. He received the Elek.Ing. (cum laude) degree from the Eindhoven University of Technology, Eindhoven, The Netherlands, in 1971.

$\mathrm{He}$ joined the Dutch Foundation for Fundamental Research on Matter (FOM) Laboratory on Plasma Physics, Rijnhuizen. The Netherlands, as a Researcher. In 1973, he moved to the Philips Research Laboratories to start research in the area of optical fiber communication systems. In 1983, he was appointed part-time Professor at Eindhoven University of Technology. He became a Full Professor in 1994, and is currently Chairman of the Department of Telecommunication Technology and Electromagnetics (TTE). Most of his work has been devoted to single-mode fiber systems and components. Currently, his research programs are centered on ultrafast all-optical signal processing, high-capacity transport systems, and systems in the environment of the users. He has more than 40 patents and has authored and co-authored more than 100 papers, invited papers, and chapters in books. In Europe, he is closely involved in Research Programs of the European Community and in Dutch national research programs. He is one of the founders of the Dutch COBRA University Research Institute. In 2001, he brought four groups together to start a new international alliance called the European Institute on Telecommunication Technologies (eiTT).

Dr. Khoe is an Associate Editor of the IEEE JOURNAL OF QUANTUM ELECTRONICS. His activities include many conferences, where he has served in Technical Committees, Management Committees, and Advisory Committees as a Member or Chairman. Recently, he was General Co-Chair of the ECOC 2001. He has been involved in journal activities as an Associate Editor, as a member of the Advisory Board, or as Reviewer. He has served the IEEE Lasers and Electro-Optics Society (LEOS) as the European Representative in BoG, Vice President of Finance and Administration, BoG Elected Member, and as a member of the Executive Committee of the IEEE Benelux Section. He was founder of the LEOS Benelux Chapter, and is the appointed 2002 President Elect of LEOS. He received the MOC/GRIN Award in 1997 and is one of three recipients of the prestigious Top Research Institute Photonics Grant that was awarded to COBRA in 1998 by the Netherlands Ministry of Education, Culture and Science. 